\title{
EVALUATE THE PERFORMANCE OF POWER ENERGY OUTPUT FORECASTING IN PHOTOVOLTAIC CELL
}

Dr. Sreeja Mole S $S^{1}$ and Shiju Thankappan ${ }^{2}$

Abstract- This paper illustrates an adaptive approach based on Artificial Neural Network (ANN) for power energy output forecasting of photovoltaic (PV) modules. Solar energy has the greatest energy potential and PV array permit to produce electric power directly from sunlight. ANN training is performed with error Back Propagation algorithm and feed forward network is used as network structure. The output power of solar photovoltaic cell is predicted on hourly basis. The dataset was collected from GECAD photovoltaic system. The accuracy of prediction can be done by using various error measurement criteria and performance is noted.

Keywords - Energy forecasting, Error Back Propagation.

\section{INTRODUCTION}

Among Renewable Energy Sources (RES), solar energy has the greatest energy potential and PV arrays permit to produce electric power directly from sunlight; furthermore, during the operational phase, the energy production occurs without fossil-fuel consumption or noise, and not posing health and environmental hazards. These features will make the PV devices one of the most important among the technologies based on the exploitation of RES [6].

Nevertheless, the technological and environmental benefits of PV technology are hindered by economic and technical factors. The high cost of production and installation make the PV technology feasible to the customer only if there are public funding opportunities [7]. Furthermore, there are various concerns associated with PV modules, such as the impact of their interconnection to the grid. But, in general, there is little information on the topic. The most severe disturbance caused by the connection of a large amount of PV generation to the grid would be encountered when a band of cloud sweeps over an area with a large concentration of PV generators. This could result in a fairly large and sudden variation in the PV output. The stochastic input from the RES as a consequence brings problems in balancing the energy supply and demand, and, in a power market perspective, different fees and marginal prices for over- and under-generation. Some countries have already defined technical specifications for energy time scale forecasting required for power dispatching plans and operations of grid-connected PV plants. Thus, forecasting of RES power generation is vital to help grid operators better manage the electric balance between power demand and supply, and to improve the penetration of distributed renewable energy sources and, in stand-alone hybrid systems, for the optimum size of all its components and to improve the reliability of the isolated systems[10].

\footnotetext{
${ }^{1}$ Professor, Christu Jyothi Institute of Technology and Science, India

${ }^{2}$ Assistant Professor,PKCET.
} 
In order to make energy supply planning rational, forecasts of RES production have to be made on the basis of the weather conditions. Any output from the weather models must then be converted into electric energy output. Reviewed literature shows that ANN [3] methods have been successfully applied for forecasts. Soft computing techniques based on ANN are used for few-hours power output forecast. Thus in statistical approach of forecasting process, artificial neural network plays an important role photovoltaic energy forecasting. In order to define the accuracy of the prediction, some error indexes are introduced to evaluate the performances of the forecasting models.

\section{ARTIFICIAL NEURAL NETWORK}

An Artificial Neural Network (ANN) is a computational model that is inspired by the structure and functional aspects of biological neural networks. It consists of an interconnected group of artificial neurons, and it processes information using a connectionist approach to computation. In most cases, an ANN is an adaptive system that usually changes its structure based on external or internal information that flows through the network during the learning phase.

Multilayered Perceptrons [5] has been applied successfully to solve some difficult and diverse problems basing on a preliminary supervised training with error back propagation algorithm using an error correction learning rule. Basically, error back learning consists in two pass through the different layers of the network, a forward pass and backward pass. In the forward pass an activity pattern (input vector) is applied to the sensory nodes of the network, its effect propagates through the network layer by layer to produce an output as actual response. During the backward pass synaptic weights are adjusted in accordance to an error correction-rule. The error signal (subtracted from a desired value) is then propagated backward through the network against the direction of the synaptic connections.

\section{A. Feedforward network}

The feed forward neural network [4] was the first and arguably simplest type of artificial neural network devised. Feed-forward ANNs allow signals to travel one way only, from input to output. There is no feedback (loops) i.e. the output of any layer does not affect that same layer. Feed-forward ANNs tend to be straight forward networks that associate inputs with outputs.

The outputs of neurons in each layer are fed forward to their next level, until the entire output of network is obtained. It usually consists of an input layer, multiple hidden layers and an output layer[9]. Through adaptable synaptic weights, each single neuron is connected to other neurons of a previous layer. Knowledge is usually stored as a set of connection weights.

Consider a neural network with input signal to the neuron in hidden layer. Each neuron in the hidden layer sum ups its input signal after weighting them with the strengths of respective connections from the input layer and computes its output as a function of the sum. Consider a neural network with input signal

to the neuron in hidden layer. Each neuron in the hidden layer sum ups its input signal after weighting them with the strengths of respective connections from the input layer and computes its output as a function of the sum.

$Y_{i}=f \sum_{i=1}^{n} w_{i j} x_{i}$

Where f can be a simple threshold function. The output of the neuron in the output layer is computed similarly. Neural networks working process is divided into two steps: the first step is called learning (or training) process, connection weights are modified by learning, connection weights matrix changes adaptively with the external environment incentives essentially. The second step is called network operating process, connection weights are fixed at this time, and the corresponding output is obtained. 


\section{NEURAL NETWORK IN PHOTOVOLTAIC FORECASTING}

A. GECAD PV dataset

This data is obtained from GECAD photovoltaic system. The capacity of one photovoltaic panel is $200 \mathrm{~W}$. This dataset was collected for each and every five minutes time interval. This dataset was collected for one month, that is, from $1^{\text {st }}$ May 2015 to $31^{\text {st }}$ May 2015.The parameter of the dataset can be as follows,

Actual solar radiation

Sensor usage

Ambient temperature

Module temperature

Total amount of energy

There is about 8451 data contained in the respective dataset collected from GECAD photovoltaic system. Thus the dataset contains the solar radiation average which is the amount of daily irradiance from the sun.

\section{ERROR BACK PROPAGATION ALGORITHM}

The learning step is an important subject of neural networks; supervised learning and unsupervised learning are two types of learning models. The Back-Propagation (BP) [1] algorithm is one of the most powerful supervised learning algorithms. But it is discovered that BP algorithm has the following shortcomings: easily falling into local minimum by using gradient-descent algorithm to converge the error between actual output and expected output; slow convergence rate; training process prone to oscillations and so on.

The neural network training was carried out by "Error Back Propagation algorithm"[8]. The MATLAB code for the EBP is written by providing the dataset as input and target. Different activation functions are used during network training.

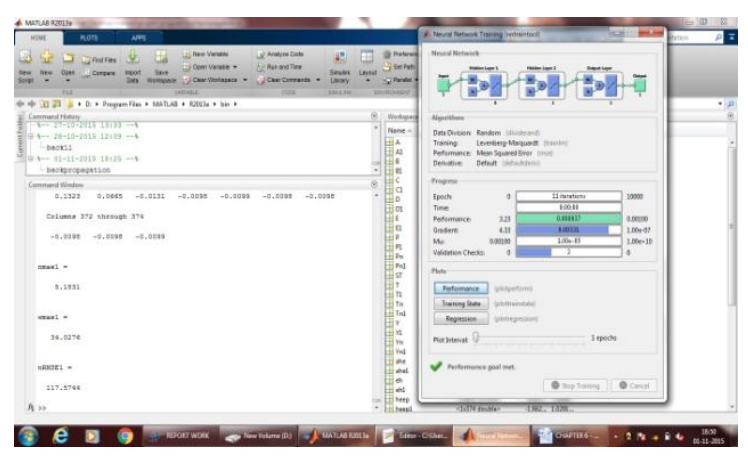

Figure1. Neural Network training by BP

The above output figure 1 was obtained by computing error Back Propagation (BP) by providing the input data (4 input) and target data (1 output) from dataset collected from GECAD PV system. 


\section{EVALUATION CRITERIA}

To test the performance of the developed model. In order to correctly define the accuracy of the prediction and to relate error, it is

necessary to define the indexes that can be used to evaluate the performance of the forecast model.

Normalized Mean Absolute Error(NMAE\%) based on net capacity of the photovoltaic panel from which the dataset is collected , $\mathrm{C}$ is the net capacity of plant[2].

Normalised Mean Absolute Error (NMAE) is based on net capacity of plant from which dataset is collected. When NMAE value is lower, then accuracy of prediction will be greater. The NMAE for GECAD dataset is 24.31 for training and 22.38 for testing. The result of NMAE is given in table 1 .

Weighted Mean Absolute Error (WMAE) is greater when NMAE is used (during unstable days). The WMAE for GECAD dataset is 4.15 for training and 3.43 for testing. The result of WMAE is given in table 1 .

Normalised Root Mean Square Error (nRMSE) - measures the average magnitude of absolute hour error. It gives higher weights to larger error. The nRMSE for GECAD dataset is 132.66 for training and 83.95 for testing. The result of nRMSE is given in table1.

These are parameter to evaluate the accuracy of prediction.

The MATLAB code for error Back Propagation (BP) algorithm with GECAD dataset (4 inputs) for Photovoltaic output power forecasting is executed and the predicted power output is compared with measured output power.

The implementation of network can be done by considering eight neurons in the hidden layer and one neuron in output layer.

Table 1. Training a Neural Network by BP algorithm, NMAE, WMAE, nRMSE for GECAD dataset

\begin{tabular}{|l|l|l|}
\hline \multicolumn{2}{|l|}{ Error } & $\begin{array}{l}\text { GECAD PV System } \\
\text { Dataset }\end{array}$ \\
\hline \multirow{4}{*}{ NM AE } & Training & 24.3166 \\
\cline { 2 - 3 } & Testing & 22.3855 \\
\hline \multirow{3}{*}{ WM AE } & Training & 4.1576 \\
\cline { 2 - 3 } & Testing & 3.4382 \\
\hline \multirow{3}{*}{ nRMSE } & Testing & 132.6648 \\
\cline { 2 - 3 } & & 83.9534 \\
\hline
\end{tabular}




\section{V1 CONCLUSION}

Photovoltaic forecasting method based on Artificial Neural Network (ANN) using error Back Propagation (BP) algorithm was done. Here GECAD PV dataset is used for analysis. The training and testing of the datasets was done with back propagation algorithm using MATLAB code and the obtained training and testing output power is tabulated and plotted. The error assessment was done to highlight the accuracy of prediction.

\section{REFERENCES}

[1] Adel Mellit, Alessandro Massi Pavan. , "Performance prediction of $20 \mathrm{kWp}$ grid-connected photovoltaic plant at Trieste (Italy) using artificial neural network", Energy Conversion and Management,Vol.51, No.12, pp.2431-2441, December 2010.

[2] M. Aghaei, A. Dolara, F. Grimaccia, S. Leva, M. Mussetta, E. Ogliari, "PV plant planning and operations by neural network analysis and validation", in 29th European Photovoltaic Solar Energy Conference and Exhibition, Milano, Italy, Vol 8, No.2, pp 978-984 September 2014.

[3] Alessandro Massi Pavan, Adel Mellit, "A 24-h forecast of solar irradiance using artificial neural network: Application for performance prediction of a grid-connected PV plant at Trieste, Italy”, Solar Energy, Vol. 84, pp.807-821, May 2010.

[4] A. Chaouachi, R.M Kamel, R. Ichikawa, H. Hayashi, and K. Nagasaka, "Neural Network Ensemble-based Solar Power Generation Short-Term Forecasting", International Journal of Electrical, Computer, Energetic, Electronic and Communication Engineering, Vol. 3, No.6, pp.1258-1263.

[5] E. Praynlin, P. Latha, "Software Cost Estimation Models using Elman Neural Network", International Journal of Information Sciences \& Computing, Vol. 7, No.1, pp. 60-65, 2013.

[6] Christophe Paoli, Cyril Voyant, Marc Muselli, Marie Laure Nivet, "Forecasting of preprocessed daily solar radiation timeseries using neural networks", Solar Energy, Elsevier, Vol.84, No.1, pp.2146-2160, November 2010.

[7] Crescenzio Gallo, Michelangelo De Bonis, "A Neural Network Model for Forecasting Photovoltaic Deployment in Italy", International Journal of Sustainable Energy and Environment, Vol.1, No.1, pp.01 -13, February 2013.

[8] E. Praynlin, P. Latha, "Software Effort Estimation Models Using Radial Basis Function Network", International Journal of Computer, Electrical, Automation, Control and Information Engineering, Vol. 8, No.1, pp. 258-263, 2014.

[9] Kumar B Mari, P Latha, E Praynlin, "Software Effect Estimation using Genetic Algorithm", International Journal on Information Sciences and Computing, Vol. 8, No.1, 2014

[10] F. Grimaccia, S. Leva, Marco Musetta, Emanuele Ogliari, “Analysis and Validtion of ANN PV power output forecasting at 24 hours ahead",Electrimacs 2014, pp. 19-22, 